\title{
Associative learning measured with ERP predicts deferred imitation using a strict observation only design in 14 to 15 month old children
}

Mikael Heimann, Emelie Nordqvist, Mary Rudner, Mikael Johansson and Magnus Lindgren

\section{Linköping University Post Print}

N.B.: When citing this work, cite the original article.

This is the author's version of the following article:

Mikael Heimann, Emelie Nordqvist, Mary Rudner, Mikael Johansson and Magnus Lindgren, Associative learning measured with ERP predicts deferred imitation using a strict observation only design in 14 to 15 month old children, 2013, Scandinavian Journal of Psychology, (54), 1, 33-40.

which has been published in final form at:

http://dx.doi.org/10.1111/sjop.12005

Copyright: Wiley-Blackwell

http://eu.wiley.com/WileyCDA/Brand/id-35.html

Postprint available at: Linköping University Electronic Press

http://urn.kb.se/resolve?urn=urn:nbn:se:liu:diva-84325 
Associative learning measured with ERP predicts deferred imitation using a strict observation only design in 14 to15 month old children

\author{
Mikael Heimann ${ }^{1,2}$, Emelie Nordqvist ${ }^{1,2}$,'Mary Rudner ${ }^{1,2}$, \\ Mikael Johansson $^{3} \&$ Magnus Lindgren ${ }^{3}$
}

1. Department of Behavioural Sciences and Learning, Linköping University

2. The Swedish Institute for Disability Research, Linköping University

3. The Department of Psychology, Lund University

Acknowledgments: The authors would like to thank all the parents and children who patiently took part in this study. We also thank Kristina Borgström and Emilia Thorup for their assistance in collecting part of the data. This research was supported by grants from the Swedish Council for Working Life and Social Research (\#2006-1040) and the Swedish Research Council (\#2011-1913) to MH. The Linnaeus Centre Thinking in Time: Cognition, Communication, and Learning financed by the Swedish Research Council (\#349-2007-8695) provided additional support.

Corresponding author; Mikael Heimann, Department of Behavioural Sciences and Learning, Linköping University, SE-58183 Linköping, Sweden. Email: mikael.heimann@liu.se 


\begin{abstract}
Deferred imitation is an established procedure for behavioural measurement of early declarative-like memories in infancy and previous work has indicated a link between this type of memory and brain potentials in infants. The present study compared infants' memory performance in this paradigm with electrophysiological indices of associative learning. Thirty children ( $\mathrm{M}=14.5$ months) participated, of which 15 (9 boys) had acceptable event-related potentials (ERP) recordings that could be included in the final analysis. Deferred imitation was measured with an observation-only procedure using three actions and a 30 min delay. ERP was recorded with a high-density electrode net (128 electrodes) during associative learning. Change scores based on Nc, a middle latency component associated with attentional processes, predicted deferred imitation performance. Thus, associative learning measured with ERP predicts deferred imitation using a strict observation only design in 14 to 15 month old children.
\end{abstract}


Associative learning measured with ERP predicts deferred imitation using a strict observation only design in 14 to 15 month old children

Long-term memory is a prerequisite for learning and cultural socialization. Up to quite recently, learning in early infancy was assumed to be based solely on implicit memory while explicit memory was assumed to show a protracted development. However, observations from the last two decades of research strongly suggest a much narrower developmental gap between implicit and explicit memory. It has even been suggested that no such gap exists, and that explicit and implicit memory capacities might develop in parallel from early on (e.g.: Howe, 2011; Rovee-Collier, Hayne \& Colombo, 2001). This new knowledge about early memory development has mostly emerged from studies that have used one of three dominating tasks/paradigms: visual paired comparison, the mobile conjugate reinforcement paradigm or deferred imitation. In this paper, deferred imitation is in focus.

Deferred imitation (DI) is a nonverbal measure of explicit memory based on the ability of infants to perform specific actions with objects after the target action has been demonstrated. Successful performance requires that the child can form a representation of a seen action, store that representation in memory and retrieve it at a later point in time. The method has been used in numerous studies that have revealed remarkable memory abilities in the first 18 months of life (for reviews see Jones \& Herbert; 2006 or Richmond \& Nelson, 2007). Presently, researchers using deferred imitation to investigate memory in infancy adopt two different methods. One is a strictly observation-only procedure (Meltzoff, 1988a) in which the child is only allowed to watch the target acts being modeled during a brief presentation. No verbal cues are given either during modeling or at the time of retrieval. The other method, elicited imitation, allows the child to spontaneously explore the objects before modeling in order to create a baseline (Bauer, 2002; Bauer, San Souci \& Pathman, 2010) and often also adds verbal support. Although the results from these two designs often seem to 
converge there are also definite non-trivial differences. One such example is the retention time. Infants tend to remember for longer periods if they are given the opportunity to explore the objects used before the target actions are demonstrated or if an immediate imitation test is carried out before a delay period is imposed (Oakes \& Bauer, 2007). In addition, the use of verbal support or verbal cues also affects the retention length (Hayne \& Herbert, 2004). Based on these differences, Howe (2011, p. 21) raises the possibility that the elicited imitation paradigm "may artificially inflate memory". Thus we need to retain the distinction between these two procedures. The DI paradigm employed in the present study is motivated by previous studies that have used the observation-only design. This was also the method used by the initial studies that gradually lowered the age boundary for the ability to perform deferred imitation tasks from 18 months as proposed by Piaget first to 14, then 9 and now to 6 months (Barr, Dowden \& Hayne, 1996; Heimann \& Meltzoff, 1996; Heimann \& Nilheim, 2004; Meltzoff, 1988a,b). Infant memory researchers agree that observation-only deferred imitation is a robust, easily administered and reliable nonverbal method of capturing rudimentary declarative memory function prior to the onset of productive language (Lukowski et al., 2005; Rovee-Collier, Hayne \& Columbo, 2001). Although evidence from studies on clinical populations suffering from temporal lobe amnesia or developmental amnesia indicate that the deferred imitation procedure taps explicit memory much detailed knowledge is still lacking (Adlam, Vargha-Khadem, Mishkin \& de Haan, 2006; McDonough, Mandler, McKee \& Squire, 1995; Richmond \& Nelson, 2007). We do not understand exactly how brain maturation is related to early memory development. Further, we have little or no knowledge of early individual differences in memory and what they might indicate for concurrent or long-term memory performance.

Individual differences in behavioral memory performance have been reported both by studies using the elicited imitation procedure (e.g. Bauer, 2002) and studies using the 
observation-only design (Heimann \& Meltzoff, 1996). For the latter method, both a concurrent correlation between deferred imitation and communication at 14 months and a predictive correlation from DI at 9 months to gestural communication at 14 months have been reported (Heimann et al., 2006). A follow-up revealed that, together with joint attention, deferred imitation also predicted cognition at 4 years of age (Strid et al., 2006).

In order to achieve a better understanding of the observed variation in memory performance between infants as reflected by the deferred imitation procedure, researchers have been motivated to use different brain measures, foremost electroencephalography (EEG) and event-related potentials (ERPs; Cycowicz, 2000; Reynolds, Guy \& Zangh, 2011; Riggins, Neely, Bauer, Georgieff \& Nelson, 2009). ERP is an EEG-derived signal reflecting the sum of large numbers of neurons firing in synchrony in response to a specific stimulus (de Haan, 2007). It is a non-invasive method making it possible to capture brain activity during cognitive tasks (e.g. memory encoding or recall) even in pre-verbal infants and a large literature has identified cognitively relevant components that can be indexed by amplitude and latency (e.g. DeBoer, Scott \& Nelson, 2005; Thomas \& Casey, 2003).

There are at present only a few studies that have used electrophysiology to explore individual differences in early memory performance based on the DI paradigm (see Richmond $\&$ Nelson, 2007 for a review). All have used the elicited imitation procedure, that is, the child has been allowed initially to explore the objects in order to create a baseline measure (thus possible inducing a motor memory). In addition, a narrative has often been added guiding the child verbally through the procedure. No study has, to our knowledge, combined Meltzoff's observation-only procedure (without being allowed to handle the objects before the task is presented and no narration) directly with measures of ERP (thus the child has to rely on an event memory based solely on observation). The findings to date, based on elicited imitation studies, suggest that both mid- and long-latency components are related to early memory 
processes in infancy (Richmond \& Nelson, 2007), although the exact latencies also varies with the age of the child. As an example, Webb, Long and Nelson (2005) demonstrated that the latency for observing $\mathrm{Nc}$, a mid-latency reflecting attentional processes, decreases from over $600 \mathrm{~ms}$ at four months of age to about $500 \mathrm{~ms}$ at 12 months.

In an early study, Carver, Bauer and Nelson (2000) used deferred imitation and ERP to study memory over a one-month delay in nine to ten months old children. The sequence of actions to be learned was modeled several times over three consecutive days. In addition, the children were allowed to handle the objects on day one in order to establish a baseline. Eventrelated-potentials reflecting recognition memory (pictures of old and new behavioral actions on objects were used as stimuli) was measured one week after the last demonstration of the target actions (= day 3 ) while behavioral recall was observed one month after modeling. In short, the study revealed (i) a stronger fronto-central mid-latency $(\mathrm{Nc})$ response (a window of 260 to $870 \mathrm{~ms}$ was used and a peak was observed at about $520 \mathrm{~ms}$ ) to new events than to old, possibly reflecting allocation of attention (Richards, Reynolds \& Courage, 2010), (ii) a negative late slow wave (SW) measured over three midline leads (Pz, Cz, and Fz) that probably reflect novelty detection according to Carver et al. (2000), and (iii) a positive slow wave indicating updating of memory in response to the old stimuli. More importantly, the Nc and the slow waves were only evident among those children who had demonstrated memory through their behavioral response. These findings have since been replicated and extended in two subsequent studies. Bauer et al. (2003) could confirm that the Nc did reflect individual differences in memory performance although in this study it was the latency and not the amplitude that was predictive. The latency to peak amplitude was longer for familiar sequences than for new. Further support was gathered by Bauer et al. (2006) who demonstrated age-related differences in responses to old and new sequences when comparing 9- and 10-month old children. As an example, a significant correlation was observed between 
immediate recognition as measured by ERP and behavioral recall one month later at 10 months but not when the children were 9 months old. Moreover, Morasch and Bell (2009) investigated to what degree EEG (electroencephalography) measured continuously over baseline, encoding and retrieval could be related to behavioral performance over a 24-hour delay in a group of 10 month olds. They examined EEG continuously and found an increase in 6-9 $\mathrm{Hz}$ EEG power at anterior temporal regions during recall as compared to baseline, but only for those children having displayed ordered-recall. In sum, all studies presented so far that have used the elicited imitation procedure report that electrophysiological indices of recognition are associated with behavioral recall.

The main goal of the present study is to explore the relationship between association memory measured in an ERP-procedure and non-verbal recall memory observed behaviorally in a deferred imitation design. For this purpose, we developed an ERP procedure inspired by the association-learning paradigm used by Torkildsen et al. (2009) for studying word learning in 20 months old children. In this procedure, the infants learn associations between pairs of novel pictures, one depicting an animal and one showing an object. During the training/learning phase links between the two stimuli being presented together are created through repeated co-presentations. In the test phase that follows directly after the end of the training phase, two alternative pairs are presented: Either a novel combination (one picture is known and one depicts a new object) or a recombination (previously shown pairs are recombined into new pairs although each individual picture is familiar).

Since deferred imitation in part relies on the ability to memorize an association between an action and a specific outcome we planned to compare ERP measured in our associative learning paradigm with behavioral tests of deferred imitation using a strict observation only design (Heimann \& Meltzoff, 1996). That is, the children were not allowed to explore the toys beforehand and no narration took place during the deferred imitation procedure; the children 
only saw the three actions to be memorized under a brief presentation phase, each action being presented three times over 20 seconds. Thus, the objects were unknown to the child before demonstration of the actions. The procedure closely matched that used previously in purely behavioral studies (Heimann et al., 2006; Strid et al., 2006), the main difference being that instead of measuring visual recognition memory during the delay, our ERP associative learning paradigm was measured instead. Thus, we measured both behavioral responses reflecting recall from memory and electrophysiological responses to associative learning association within a 30-minute time frame. We reasoned that deferred imitation, besides being indicative of early representational abilities (Meltzoff, 2004) also requires the child to associate an action with an outcome and that the ERP association learning paradigm to some degree taps similar learning processes. On the basis of the previous literature on measuring ERP in infancy in general (e.g.: Handy, 2005; de Haan, 2007) and on deferred imitation in particular (e..g: Carver et al., 2000) we predicted an amplitude decrease in the Nc response as association pairs were repeated and learned. More specifically, we predicted a significant difference between the observed amplitude at the end of the training phase in the ERP procedure and measured amplitudes when a changed stimulus is presented to the child (a recombination of known stimuli or a known stimulus paired with a novel stimulus). Furthermore, we also suspected that these differences in amplitude would correlate with behavioral indices of learning as evident from the children's deferred imitation performance. Since our assumption was that associative learning serves as a bridge between electrophysiological and behavioral measures of memory, we especially expected that the difference in amplitude between the training phase and the recombination of previously learned stimuli would predict memory as measured with deferred imitation, Finally, we also explored a possible relationship to a late slow wave as reported by Carver et al. (2000). 


\section{Participants}

The initial sample included 30 infants aged fourteen to fifteen months $(\mathrm{M}=14.47 ; \mathrm{SD}=$ 0.87 ; range 13-16; 17 boys and 13 girls) with a mean birth weight of 3671 grams (SD = 465.1), a mean Apgar score at five minutes postpartum of $9.67(\mathrm{SD}=0.73)$ and a mean gestational age of 39.9 weeks $(\mathrm{SD}=1.58$; range $37-42)$. Fifteen participants were excluded due to absence or unreliability of ERP data (see below for details). Thus, this report includes data from the remaining 15 children ( 8 boys and 7 girls) with a mean age of 14.30 months $(\mathrm{SD}=0.74$; range $13.1-15.7 ; 95 \% \mathrm{CI}: 13.9-14.7)$. The children were recruited through well-baby clinics, open day-care units for parents and their infants, and word of mouth. The socioeconomic status was middle to upper class based on information of the parents' educational level.

\section{Procedure}

All children were tested either at Linköping University $(\mathrm{n}=17)$ or Lund University $(n=13)$, Sweden. Both sites had fully equipped laboratories using similar hard- and software for measuring electrophysiology and behavior. The study was approved by the Regional Ethical Review Board, Linköping University.

\section{Behavioral measure of memory}

Deferred imitation. The procedure implemented was similar to that used in two previous studies (Heimann \& Meltzoff, 1996; Heimann et al., 2006). A set of three toys identical to the toys reported as age appropriate by Meltzoff (1988a) was used. Each toy and its associated action were presented three times, one toy at a time, and the order of presentation of the different toys was cross-balanced across subjects. The child sat in the parent's lap during the presentation and performance of the tasks. A strict observation only design was used, that is, the child was not allowed to touch the toy either during the presentation or immediately after; thus no baseline was used. The presentation length was 
approximately $20 \mathrm{~s}$ for each toy; the experimenter adapted the presentation so that the child was always visually attending to the action presented. No verbal description of the tasks was given except for attempts to keep the child's attention, e.g.: "Look at this!" or "Look what I am doing".

The first object was a pull toy that could be pulled apart and put back together again. It consisted of two white plastic cylinders, each with a $7.5 \mathrm{~cm}$ length of plastic tubing extending from it. One length of tubing was slightly narrower and fitted inside the other. The action demonstrated was to pick up the object by the ends and pull outward with a very definite motion so that the toy came apart. The second object was a collapsible cup that could be folded up like a telescope by pressing downward on it. The action demonstrated was to show the cup in its unfolded position and press it closed using the open palm on the top surface. The third action used two objects: One empty plastic cup and a string of beads. The action demonstrated was to pick up the beads and slowly lower them into the plastic cup. A break followed the presentations, during which children and parents were taken to the laboratory for the electrophysiological recording. The imposed delay was approximately 30 minutes long (M $=26.63 ; \mathrm{SD}=7.35 ;$ range 18 to 40 ). After completion of the ERP procedure the child was taken back to the presentation room and invited to use the toys one by one.

\section{Electrophysiological measures of memory}

Participants sat on a parent's lap and visual stimuli were displayed on a 30 x $40 \mathrm{~cm}$ computer monitor placed approximately $1 \mathrm{~m}$ in front of participants. Participants were videomonitored during the whole electrophysiological session that lasted for approximately 30 minutes including introduction to the lab environment, capping, and impedance measures. The actual ERP-recording lasted approximately 10 minutes (range 9-13).

Stimulus material for measuring ERP. Stimuli were presented in blocks that employed four pairs of pictures (color clip art). In each pair one picture depicted an animal (e.g. dog) 
that was associated with an inanimate object (e.g. car). The blocks were divided into a learning phase (familiarization) and a test phase. In the learning phase, two animals and two objects were paired (e.g. dog-car and deer-house) and each association was presented five times (Presentation $1-5$ ); thus each complete learning phase included a total of ten presentations (five of each pair to be learned). Each presentation lasted $1500 \mathrm{~ms}$ with an interstimulus interval that was $1000 \mathrm{~ms}$ or longer since each stimulus pair was presented manually. The child's attention was monitored via a live video signal and a new stimulus was only presented if the child was looking at the screen. Presentations of the two stimulus-pairs included in the learning phase were not randomized but the same pair never appeared twice consecutively. Thus, there were one intervening trial and a minimum of $3500 \mathrm{~ms}$ between each presentation of the same picture pair. During the pause between each stimulus pair an attention grabber was displayed for $1000 \mathrm{~ms}$ : A clown was shown and a voice was heard over the speakers saying, "Look now" ("Titta nu"). The test phase of the block consisted of two incongruity conditions where a previously shown stimulus was associated with the 'wrong' object, i.e. a stimulus with which it had not been paired previously. In one of the incongruity trials (recombined) the associations learned were switched so that previously learned associations were violated (e.g.: if dog-car and deer-house was presented during the learning phase, the recombined incongruity trials presented dog-house and deer-car). In the second type of incongruity trials two completely new associations (novel object) were presented (e.g. dog-ball and deer-umbrella). In addition, a reminder (presentation 6) of each of the pairs presented during the learning phase was also shown during the test phase. The reminder and the incongruity trials were presented twice and the order was pseudo-randomized so that a reminder never appeared directly after the last stimulus pair in the learning phase. There was no marker that indicated where the learning phase ended and the test phase of the block began. Results from both the learning and test phases of the experiment are reported. 
Recording of ERP. The EEG was recorded with the Electrical Geodesics HydroCel High Density sensor net consisting of 128 electrodes, where no abrasion of the skin is necessary due to the connection to a high-impedance amplifier (the Net Amps 300). The vertex was used as reference and was subsequently re-referenced offline to average reference used for analysis. Impedances were kept below $50 \mathrm{k} \Omega$ and the gain was set to 20,000. Filters were set to $0.5-30 \mathrm{~Hz}$ bandpass and the sampling rate was $250 \mathrm{~Hz}$. Data was segmented into epochs of $1500 \mathrm{~ms}$ with a pre-stimulus baseline of $100 \mathrm{~ms}$. Baseline correction was performed. Artifact detection was set to accept a max-min amplitude difference of $150 \mu \mathrm{V}$. To correct for eye blink artifacts, an eye blink correction algorithm was employed (Gratton, Coles \& Donchin, 1983). Bad channels were replaced using spherical spines as the interpolation method in accordance with the procedure specified in the Net Station manual (Electrical Geodesic, 2006). As mentioned above, each block was made up of a learning phase (10 stimuli presentations) and one test phase ( 6 presentations) and the number of blocks needed in order to be included in the analysis was approximately $10(\mathrm{M}=10.94, \mathrm{SE}=.84$; 95\% CI: $9.03-12.84)$.

\section{Attrition}

$E R P$. Fifteen children were excluded from the initial sample of 30 due to illness $(\mathrm{n}=1)$, technical errors $(n=2)$, too many artifacts or responses that exceeded $150 \mu V(n=3)$, unwillingness to wear the net $(n=3)$ or providing fewer than 10 artifact-free trials due to fuzziness or tiredness $(n=6)$. An additional child was defined as an outlier $(>3$ SD from the mean) for the reminder only (presentation 6) during the ERP learning phase.

Deferred imitation. Data from all 15 children constituting the final sample is included. Analysis of behavioral data

Deferred imitation. The scoring procedures and operational definitions were identical to the ones used in previous studies (e.g.: Heimann \& Meltzoff, 1996). The scorers viewed an 
edited and digitized version of the tape that only included the response periods. A dichotomous yes/no code was used as an indication of whether the infant produced the target action or not. A 'yes' was coded for the pull toy if the child succeeded in separating the two parts of the toy, a 'yes' for the collapsible cup was noted if the cup was completely folded, and a 'yes' for the beads and cup indicated that the string of beads ended up within the cup with no more than a third of the beads hanging over the edge of the cup. If the target action was produced within $30 \mathrm{~s}$ from when the child first touched the object, a score of one was awarded. Thus, the possible score ranged from zero to three, one point for each of the three target actions presented. Two persons, one experienced researcher and one research assistant, coded all deferred imitation tasks. The Pearson product-moment correlation assessing their agreement was $r=.91$ and Cohen's kappa $\kappa=.83$.

\section{Analysis of electrophysiological data}

$N c$ : The first step in the analysis was to identify the negative central component $(\mathrm{Nc}), \mathrm{a}$ response usually occurring at around 300-600 ms following stimulus onset. The Nc component is assumed to reflect the allocation of attention to a new or salient stimulus, and is maximal over fronto-central leads (Richards et al., 2010; Stets \& Reid, 2011). The peak amplitude within a selected time window of 300-600 ms following stimulus onset was analyzed for an average of ten leads around the Fz lead (see red markings in Fig. 1).

\section{Insert Figure 1 About Here}

Repeated measures of variance (ANOVA) and Student's t-test were used to analyze the data, i.e the difference in mean amplitudes between the learning phase and the test phase within each condition. A cluster of mid-frontal leads was used for identifying the $\mathrm{Nc}$ response. In order to conduct planned contrasts that could test our a priori hypotheses a measure of associative learning based on change scores was created (the observed difference between the incongruity trials and the presentation 5, the last presentation of the learning 
phase). Thus, change scores in mean amplitude were calculated between the last presentation in the learning phase (presentation 5) (where the participants were expected to have learned the associations and thus show a decrease of activation in the Nc component), and the two incongruity conditions in the test phase (recombined and novel object); where the Nc was expected to increase again due to the renewed attention these incongruity tasks attract. The relationship between the observed change scores and the behavioral measures (deferred imitation and vocabulary) was analyzed with Pearson product-moment correlation coefficients.

Slow waves. The second step included a more explorative analysis of possible slow waves (SW), a long-latency activity often occurring at temporal sites around 700-1500 ms following stimulus onset. These late waves have been related to several memory processes, e.g. consolidation and updating (de Haan, 2007, Reynolds, Guy \& Zangh, 2011). Here, the mean amplitude for two clusters of leads within two selected time windows ("Early"; 700$1100 \mathrm{~ms}$ following stimulus onset, and "Late"; 1100-1500 ms following stimulus onset) at both hemispheres was used for analysis. The two clusters of leads on each hemisphere were concentrated around temporal leads in the 10-20 system; T3, T4, T5, T6 (see blue markings in Fig. 1). Mean amplitudes for each cluster and time window were calculated. Repeated measures analysis of variance was conducted with condition (Learning phase and test phase), lead (mean amplitude for "lead clusters" around T3, T4, T5, T6), hemisphere (right vs left) and time window ("Early" vs "Late") as within subjects factors. Greenhouse-Geisser correction where applied when sphericity assumptions were violated. Sidak correction was used when exploring main and interaction effects.

Results

\section{Electrophysiological measures of memory}


Overall: A negative deflection (Nc) was identified over fronto-central leads within 300$600 \mathrm{~ms}$ post stimuli with a peak at about $470 \mathrm{~ms}$ (see Figure 2 for Nc measured at the Fz). In contrast, an exploratory analysis of possible slow waves within an early (700 to $1100 \mathrm{~ms}$ ) or late (1100 to $1500 \mathrm{~ms}$ post stimuli) time window revealed no significant main effect or interactions involving the factor Condition (Fs $<1.5$, ns; see Figure 1). Thus, we performed no further analyses based on slow wave data.

Learning Phase: For the learning phase the grand mean amplitudes varied from -11.68 $\mu \mathrm{V}(\mathrm{SD}=8.19$; table 1$)$ during the second presentation within a block to $-9.71 \mu \mathrm{V}(\mathrm{SD}=$ 10.96) at the last presentation before the test phase (fifth presentation; see table 1) and -8.18 $\mu \mathrm{V}(\mathrm{SD}=5.21)$ during the reminder (the sixth presentation). A GLM analysis excluding the outlier identified for presentation 6 revealed a significant linear within-subjects contrast $(\mathrm{F}[1,13]=4.78 ; p=.048)$ but a non-significant within-subjects effect $(\mathrm{F}[3,11]=2.04 ; p=$ .124). Planned comparisons between the initial learning phase (LP $1+2=$ presentations 1 and 2) and the last learning trial (presentation 5) together with the reminder (presentation 6) showed a significant decrease in amplitude $(\mathrm{t}[13]=-2.19 ; p=.048)$.

Test phase: During the test phase, the grand mean amplitudes during the two incongruity conditions were $-13.87 \mu \mathrm{V}(\mathrm{SD}=10.90)$ for the recombined and $-10.47 \mu \mathrm{V}(\mathrm{SD}=$ 9.45) for the novel object stimuli. A GLM analysis based on all 15 children including presentations 1 - 6 and the two test phases (see Figure 3) revealed a significant quadratic within-subjects contrast $(\mathrm{F}[1,14]=6.75 ; p=.021)$ but a non-significant within-subjects effect $(\mathrm{F}[3,12]=1.19 ; p=.336)$. We analyzed the result separately for the two test conditions (recombined and novel object) in order to see if a change in amplitude could be detected from the end of the learning phase (presentations 5 and 6) and the test phase in focus. An ANOVA including three conditions (presentation 5, presentation 6 and the recombined test phase) revealed a significant effect, $\mathrm{F}(2,13)=3.70 ; p=.039, \eta^{2}=.21$. Planned comparisons 
identified significant effects for the comparisons between the recombined condition and the last trial of the pure learning phase (presentation 5: $\mathrm{t}[14]=2.52, p=.024$ ) as well as for the reminder (presentation $6 ; \mathrm{t}[14]=2.37, p=.033$ ). A similar analysis focusing on the novel object combination in the test phase failed to find a significant overall effect, $\mathrm{F}(2,13)=.27 ; p$ $>.50$.

Insert Table 1, Figures 2 and 3 About Here

\section{Behavioral measure of memory}

Deferred imitation (DI). The children remembered almost two of the actions on average $(\mathrm{M}=1.87 ; \mathrm{SD}=1.06$; range $0-3)$, which is comparable with our previous studies that included a separate control group: Heimann and Meltzoff (1996) reported a mean of 1.89 (SD $=0.89)$ and Heimann et al. $(2006)$ a mean of $1.97(\mathrm{SD}=0.72)$.

\section{Relationships between electrophysiological and behavioral measures}

Nc and memory: Observed memory performance on the deferred imitation task revealed a positive correlation with the Nc change score reflecting the change in mean amplitude between the recombined condition in the test phase and the last presentation during the learning phase, $r(15)=.52 ; p<.05$ (Table 2$)$. The recombined stimuli presented the child with an associative violation, a picture of an animal were associated with the 'wrong' picture of an object. Thus, the observed change in amplitude as a response to this incongruity was linked to the children's memory performance in the deferred imitation task. This was further underscored by a median split $(\mathrm{Md}=-5.58)$ based on the $\mathrm{Nc}$ change score between the recombined condition and the last presentation of the learning phase. This resulted in two groups, one with both positive or negative amplitude change (Group A: $\mathrm{M}=0.53$; $\mathrm{SD}=5.46$; range $-5.58-7.61 ; \mathrm{n}=8$ ) and one with only negative change in amplitude (Group B: $\mathrm{M}=$ 10.15; $\mathrm{SD}=4.70 ;$ range $-18.91--5.62 ; \mathrm{n}=7$ ). The two groups differed significantly in 
behavioral memory performance as evident from their deferred imitation performance $(\mathrm{M}=$ 2.38; $\mathrm{SD}=0.92$ for Group $\mathrm{A} ; \mathrm{M}=1.29 ; \mathrm{SD}=0.95$ for Group $\mathrm{B} ; \mathrm{t}[13]=2.26 ; p=.042 ; d=$ 1.16).

\section{Insert Table 2 About Here}

\section{Discussion}

Our first prediction was confirmed in that a decrease in amplitude was observed 300 to $600 \mathrm{~ms}$ post-stimuli indicating that the children did learn the stimulus pairs presented during the implemented association-learning paradigm. The temporal characteristics of our repetition design led us to interpret this peak as an Nc (Webb et al., 2005). This response was significant for stimulus pairs recombined in new ways (recombined) but not when a completely new stimulus was introduced (novel object). One possible reason for this might be that the children reacted more to a reshuffling of learned associations than to adding a completely novel picture to a familiar one (i.e. novel object). This, partly counterintuitive finding, implies that the children allocated their attention differently in the two test conditions. More attention was allocated to a breach of a well-learned association (the recombined stimuli) than to the introduction of a new combination (novel object). Maybe the attentional system of the young infant at some times becomes more focused on minor deviances while other, to us more strong violations, go relatively unnoticed. An alternative explanation to the observed results would be that the two conditions - recombined and novel object - were in some unknown way differentially difficult and that too few trials were included in order to detect a significant effect for the novel object stimulus.

Our second, and in our view probably the theoretically most interesting prediction was also confirmed in that a positive relationship between electrophysiological and behavioral memory measures was detected. More specifically, a significant correlation was found between the Nc change score (that is, the difference in observed amplitude between the end of 
the learning phase and a presentation of a recombined stimulus in the test phase) and memory performance observed with the deferred imitation paradigm. Thus, variability in behavioral recall after a 30-minute delay at 14 months was related to brain measures that most likely reflect attentional processes (Richmond \& Nelson, 2007). A post-hoc median split based on the Nc change score further illustrated this finding: The two groups such identified recalled the actions in the deferred imitation procedure differently. The group with a slightly more negative change score showed an impoverished recall of the three memory tasks used in the deferred imitation paradigm compared to the children in the other group. The difference in performance between the two groups was significant and the effect size was large.

The children who remembered more of the action-on-object associations in the deferred imitation procedure were also the ones with the largest change in amplitude between presentation 5 and the recombined stimulus. Thus, the children displaying strong learning of the associations in the ERP procedure also displayed better explicit memory as reflected by their performance in the deferred imitation procedure. One possible reason for this might be that these children encoded more robust representations of the actions because they were faster in allocating their attention to relevant features and thus were faster to build stronger associations between observed actions and their objects. In contrast to the change score between presentation 5, the last presentation before the test phase, and the recombined test stimulus, the change score between presentation 5 and the novel object test stimulus did not relate to behavioral memory measures, there was in fact no significant difference in amplitude between presentation 5 and the novel object stimulus.

We also explored if slow waves occurring 800 to $1500 \mathrm{~ms}$ post-stimuli were related to memory performance as suggested by Carver, Bauer and Nelson (2000). This analysis failed to identify any significant relation between early or late slow waves and behavioral memory performance. This is somewhat surprising since slow waves have been linked to both novelty 
detection and updating (de Haan, 2007) and since studies using an alternate method for studying deferred imitation - elicited imitation - have been able to demonstrate links between slow waves and behavioral recall. However, elicited imitation differs in central aspects from the behavioral method used here in that the children are more familiar with the objects since they are allowed to handle them during baseline. In some studies, elicited imitation also includes both practice and narration. In addition, Carver and al. used a longer delay and an ERP procedure reflecting how the children recognized the actions presented to them (the children saw photos depicting the end state of the action or photos of actions never presented).

This is the first study having demonstrated a link between electrophysiology and behavioral measures of declarative-like memories in early infancy using a very brief presentation, allowing no exploration beforehand and no verbal support. However, the link is only indirect in that two different learning procedures were coupled together. In deferred imitation, the child had to attend to an object, note and associate the specific action carried out with that object and, finally, store this observation in memory for later use. In the ERP procedure the child was given the opportunity to associate two pictures (one animal and one object) over a series of trials after which new pairs of pictures were presented. The new pairs either included two known pictures combined in a new way (recombination) or the pairing of a new object with a previously presented picture of an animal (novel object). The link between electrophysiology and behavior needs to be investigated in more detail in further studies, possibly also using other brain measures besides such as near infrared spectroscopy (NIRS) or EEG.

There are obvious limitations with the findings presented. Most important, the large attrition is problematic although not unusual for studies using ERP with small children. Of 30 children, acceptable electrophysiological recordings could only be obtained from 15 participants. In other words, data from half of the participants were lost. This affects the 
power of the study and raises the question if the children whose data were lost in some way differed from those whose data was retained. An inspection of the available background data did not however reveal any systematic differences between the 15 children who were included and those who were not. Moreover, one reason for the lack of observed correlations between SW and deferred imitation could be that neck and temporal areas of small children where SW electrodes are located tend to yield unreliable data due to motion artifacts (rubbing of back of the head causing the electrodes to move in the temporal area).

Another limitation that might weaken our findings is the number of trials forming the basis for the analysis presented. Each block included ten learning trials and the criterion was set to ten usable blocks (that equals a total of 100 trials in the learning phase and 40 in the test trials). Most guidelines for conducting ERP-research (e.g. Luck, 2005) build on adult populations and recommend that the researcher collect more trials than was done in our study. However, this is usually not possible when studying an infant population, which has been pointed out by Stets and Reid (2011). They reanalyzed previously published materials and found that adding artifact-free trials occurring early could increase the power. This was true even if only few acceptable blocks of data had been collected for a specific subject. They suggest that "focusing the statistical analysis on fewer trials from the beginning of the experimental session in addition to examining all the artifact-free trials per condition and subject would provide new insights into infant cognitive development “ (p. 567). Thus, the limited number of blocks and trials used here does not necessarily indicate that the findings are less reliable. On the contrary, Stets and Reid point out, infant attention and the Nc response change over time and trials which means that responses recorded early and late might reflect partly different processes. Although we have not analyzed this aspect specifically, the ERP:s used in our analysis were mostly obtained from the early part of the session. 
In sum, this study has demonstrated that electrophysiological indices of learning emanating from an association paradigm predict memory recall observed behaviorally using an observation-only paradigm. Individual differences in behavioral performance on memory tasks are reflected in the observed activity of the nervous system as reflected in the $\mathrm{Nc}$ response. The Nc-response obtained by our ERP procedure seems to be sensitive to changes reflecting associative memory. The study also adds to the findings on ERP and memory reported by studies having used the elicited imitation paradigm. The two different procedures - elicited imitation and observation-only - both create robust memory traces although we still do not know to what degree they reflect similar memory processes at the neurophysiological level. Studies using both methods in parallel are still lacking. Future studies also need to use a larger sample size, explore longer delays than the 30 minutes used here, and also use other measures in order to corroborate the findings reported here. 


\section{References}

Adlam, A-L.R, Vargha-Khadem. F., Mishkin, M., \& de Haan, M. (2005). Deferred imitation of action sequences in developmental amnesia. Journal of Cognitive Neuroscience, 17 (2), 240-248.

Barr, R., Dowden, A., \& Hayne, H. (1996). Developmental changes in deferred imitation by 6- to 24-month-old infants. Infant Behavior and Development, 19(2), 159-170.

Bauer, P.J. (2002). Long-term recall memory: Behavioral and neuro-developmental changes in the first 2 years of life. Current Directions in Psychological Science, 11 (4), 137-140.

Bauer, P.J., San Souci, P., \& Pathman, T. (2010). Infant memory. Wiley Interdisciplinary Reviews: Cognitive Science, 1 (2), 267-277. DOI: 10.1002/wcs.38

Bauer, P.J., Wiebe, S.A., Carver, L.J., Waters, J.M., \& Nelson, C.A. (2003). Developments in long-term explicit memory late in the first year of life: Behavioral and electrophysiological indices. Psychological Science, 14 (6), 629-635. doi: 10.1046/j.0956-7976.2003.psci_1476.x

Bauer, P. J., Wiebe, S.A., Carver, L.J., Lukowski, A.F., Haight, J.C., Waters, J.M., \& Nelson, C.A. (2006). Electrophysiological indexes of encoding and behavioral indexes of recall: Examining relations and developmental change late in the first year of life. Developmental Neuropsychology 29 (2), 293-320.

Carver, L. J., Bauer, P. J. \& Nelson, C. A. (2000). Associations between infant brain activity and recall memory. Developmental Science, 3(2), 234-246.

Cycowicz, Y.M. (2000). Memory development and event-related brain potentials. Biological Psychology, 54, 145-174.

DeBoer, T., Scott, L.S., \& Nelson, C.A. (2005). ERPs in developmental populations. In T.C. Handy (Ed.), Event-Related Potentials: A Methods Handbook (pp. 263-298). Cambridge, MA: MIT Press. 
de Haan, M. (Ed.) (2007). Infant EEG and Event-related potentials. London: Psychology Press.

Electrical Geodesic Inc. (2006). Net Station Waveform Tools: Technical Manual. Eugene, OR: Author.

Gratton, G., Coles, M.G.H., \& Donchin, E. (1983). A new method for off-line removal of ocular artifact. Electorencephalography and Clinical Neurophysiology, 55 (4). 468-484. Doi: 10.1016/0013-4694(83)90135-9

Handy, T.C. (2005). (Ed.), Event-related potentials: A methods handbook. Cambridge, Mass: MIT Press.

Hayne, H., \& Herbert, J. (2004). Verbal cues facilitate memory retrieval during infancy, Journal of Experimental Child Psychology, 89 (2), 127-139. http://dx.doi.org/10.1016/j.jecp.2004.06.002

Heimann, M., \& Meltzoff, A.N. (1996). Deferred imitation in 9-to 14-month-old infants: A longitudinal study of a Swedish sample. British Journal of Developmental Psychology, $14,55-64$.

Heimann, M., \& Nilheim, K. (2004). 6-month olds and delayed actions: An early sign of an emerging explicit memory. Cognition, Brain, Behavior, VIII (3-4), 249-254.

Heimann, M., Strid, K., Tjus, T., Smith, L., Ulvund, S.E., \& Meltzoff, A.N. (2006). Exploring the relation between memory, gestural communication, and the emergence of language in infancy: A longitudinal study. Infant and Child Development, 15 (3), 233-249.

Howe, M.L. (2011). The Nature of Early Memory. Oxford, UK: Oxford University Press. Jones, E.J.H., \& Herbert, J.S. (2006) Exploring memory in infancy: Deferred imitation and the development of declarative memory. Infant and Child Development. 15, 195-205. 
Luck, S.J. (2005). Ten simple rules for designing ERP experiments. In T.C. Handy (Ed.), Event-related Potentials: A Methods Handbook (pp. 33-55). Cambridge, MA.: MIT Press.

Lukowski, A.F., Wiebe, S.A., Haight, J.C., DeBoer, T., Nelson, C.A., \& Bauer, P.J. (2005). Forming a stable memory representation in the first year of life: Why imitation is more than child's play. Developmental Science, 8 (3), 279-298.

Meltzoff, A.N. (1988a) Infant imitation after a one-week delay: Long-term memory for novel acts and multiple stimuli. Developmental Psychology, 24, 4470-476.

Meltzoff, A.N. (1988b). Infant imitation and memory: Nine-month-olds in immediate and deferred tests. Child Development, 59, 217-25

Meltzoff, A. N. (2004). The case for developmental cognitive science: theories of people and things. In G. Bremner \& A. Slater (Eds.), Theories of Infant Development (pp. 145173). Oxford: Blackwell Publishing

McDonough, L., Mandler, .J.M., McKee, R.D., \& Squire, L.R. (1995). The deferred imitation task as a nonverbal measure of declarative memory. Proceedings of the National Academy of Sciences (USA), 92, 7580-7584.

Morasch, K.C., \& Bell, A.B. (2009). Patterns of brain-electrical activity during declarative memory performance in 10-month-old-infants. Brain and Cognition, 71, 215-222. doi:10.1016/j.bandc.2009.08.012

Oakes L.M., \& P.J. Bauer, P.J. (2007). (Eds.), Short- and long-term memory in infancy and early childhood: Taking the first steps toward remembering New York: Oxford University Press.

Richards, J.E., Reynolds, G-D., \& Courage, M.L. (2010). The neural basis of infant attention. Current Directions in Psychological Science, 19 (1), 41-46. doi: $10.1177 / 0963721409360003$ 
Riggins, T., Neely, C., Bauer, P.J., Georgieff, M.K., \& Nelson, C.A. (2009).

Electrophysiological indices of memory for temporal order in early childhood: implications for the development of recollection. Developmental Science, 12 (2), 209219. DOI: $10.1111 / \mathrm{j} .1467-7687.2008 .00757 . \mathrm{x}$

Reynolds, G.D., Guy, M.W., \& Zangh, D. (2011). Neural correlates of individual differences in infant visual attention and recognition memory. Infancy, 16 (4), 368-391. DOI: 10.1111/j.1532-7078.2010.00060.x

Richmond, J., \& Nelson, C.A. (2007). Accounting for change in declarative memory: A cognitive neuroscience perspective. Developmental Review, 27, 349-373.

Rovee-Collier, C., Hayne, H., \& Columbo, M. (2001). Development of implicit and explicit memory, (Advances in Consciousness Research, Series B, Vol. 24). New York: Benjamin.

Stets, M. \& Reid, V.M. (2011). Infant ERP amplitudes change over the course of an experimental setting: Implications for cognitive processes and methodology. Brain \& Development, 33, 558-568. Doi: 10.1016/j.braindev.s010.10.008

Strid, K., Tjus, T., Smith, L., Meltzoff, A.N., \& Heimann, M. (2006). Infant recall memory and communication predicts later cognitive development. Infant Behavior and Development, 29, 545-553.

Thomas, K.M., \& Casey, B.J. (2003). Methods for imaging the developing brain. In M. de Haan \& M.H. Johnson (Eds.), The Cognitive Neuroscience of Development (pp. 19-41). Hove, UK: Psychology Press.

Torkildsen, J.v.K., Hansen, H.F., Svangstu, J.M., Smith, L., Simonsen, H.G., Moen, I., \& Lindgren, M. (2009). Brain dynamics of word familiarization in 20-month-olds: Effects of productive vocabulary size. Brain and Language 108 (2), 73-88.

DOI:10.1016/j.bandl.2008.09.005 
Webb, S., Long, J., \& Nelson, C. (2005). A longitudinal investigation of visual event-related potentials in the first year of life. Developmental Science, 299, 577-580. DOI: 10.1111/j.1467-7687.2005.00452.x. 
Table 1

Grand mean amplitudes for the learning phase (presentation 1-5) and the test phase (reminder, recombined and novel object)..

\begin{tabular}{|c|c|c|}
\hline Condition & Mean amplitude & SD \\
\hline \multicolumn{3}{|l|}{ Learning phase } \\
\hline \multirow[t]{5}{*}{ Presentation } & -10.95 & 8.14 \\
\hline & -11.68 & 8.98 \\
\hline & -11.10 & 9.56 \\
\hline & -10.31 & 8.12 \\
\hline & -9.17 & 10.96 \\
\hline \multicolumn{3}{|l|}{ Test phase } \\
\hline Reminder $^{l}$ & -8.18 & 5.24 \\
\hline Recombined & -13.87 & 10.90 \\
\hline Novel object & -10.47 & 9.46 \\
\hline
\end{tabular}


Table 2.

(A) Change scores between the last trial in the learning phase (presentation 5) and the two incongruity trials in the test phase (recombination and novel object).

(B) Observed correlations with non-verbal explicit memory as measured with the observation-only deferred imitation procedure.

\begin{tabular}{lcc|cc}
\hline & \multicolumn{2}{c|}{ A } & \multicolumn{2}{c}{ B } \\
& \multicolumn{2}{c}{ Change score } & \multicolumn{2}{c}{ Correlation with recall } \\
& M & SD & $r$ & $p<$ \\
\hline Recombination -Pres 5 & -4.7 & 7.2 & .52 & ns \\
Novel object -Pres 5 & -1.3 & 7.5 & .11 & .05 \\
\hline
\end{tabular}


Figure legends

Figure 1

The distribution of the electrodes used analyzing the central Nc response (red markings) and slow waves on right and left hemisphere (blue markings).

Figure. 2

Mean amplitudes of Nc response for each condition. Negative is plotted upwards. Test phase stimuli: $\mathrm{RCOMB}=$ recombined; $\mathrm{NOV}$ OBJ $=$ novel object

Figure 3

Average grand mean amplitudes for the learning phase (LP) and the test phase (TP). LP $1+2=$ grand mean amplitude averaged over presentations one and two; LP 3+4 = average over presentations three and four; LP 5+6 = average over presentations 5 and $6 . \mathrm{TP}=$ grand mean amplitude averaged over the two incongruity trials in the test phase: recombined and novel object. (Note that presentation $6=$ the reminder presented in the test phase). 
ERP and Deferred Imitation 30

Figure 1

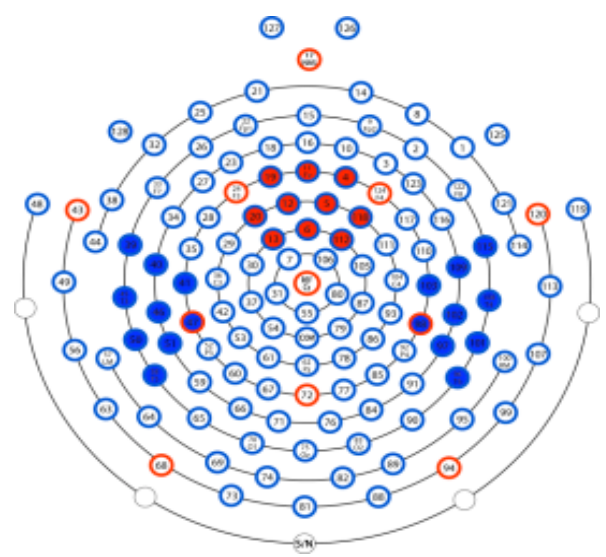


Figure 2

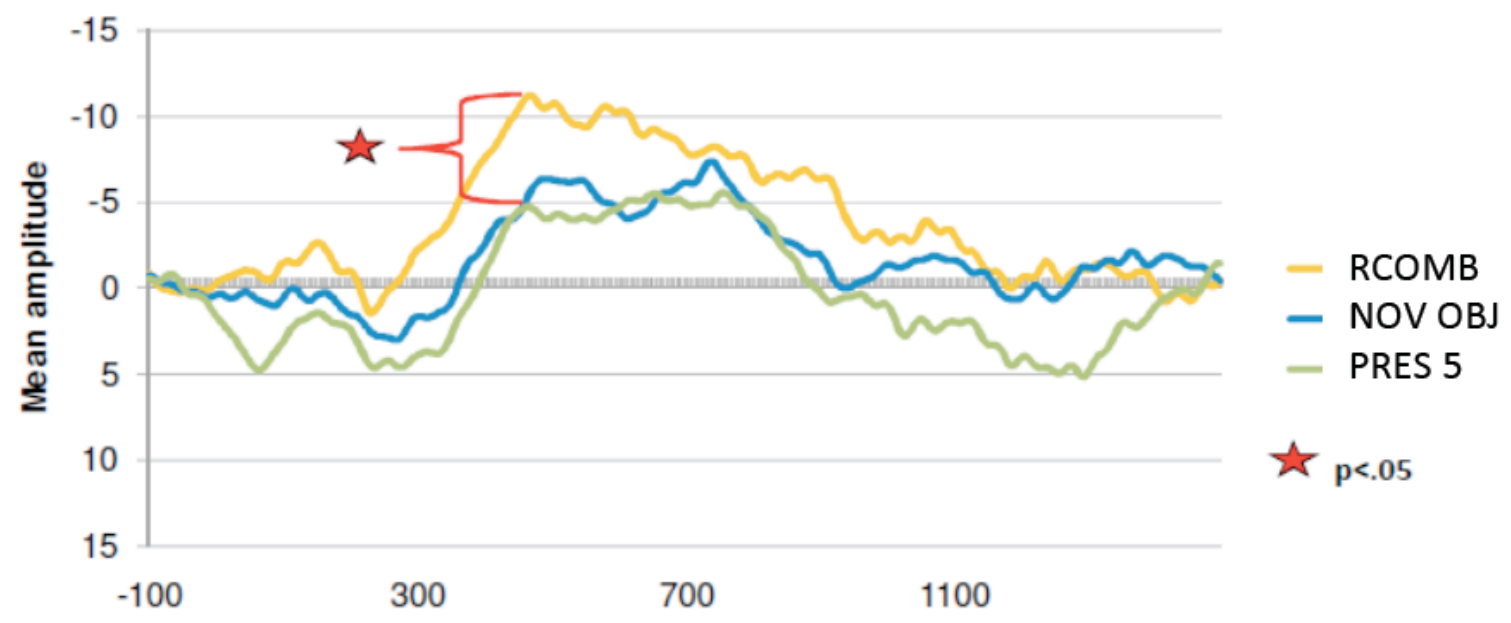


ERP and Deferred Imitation 32

Figure 3

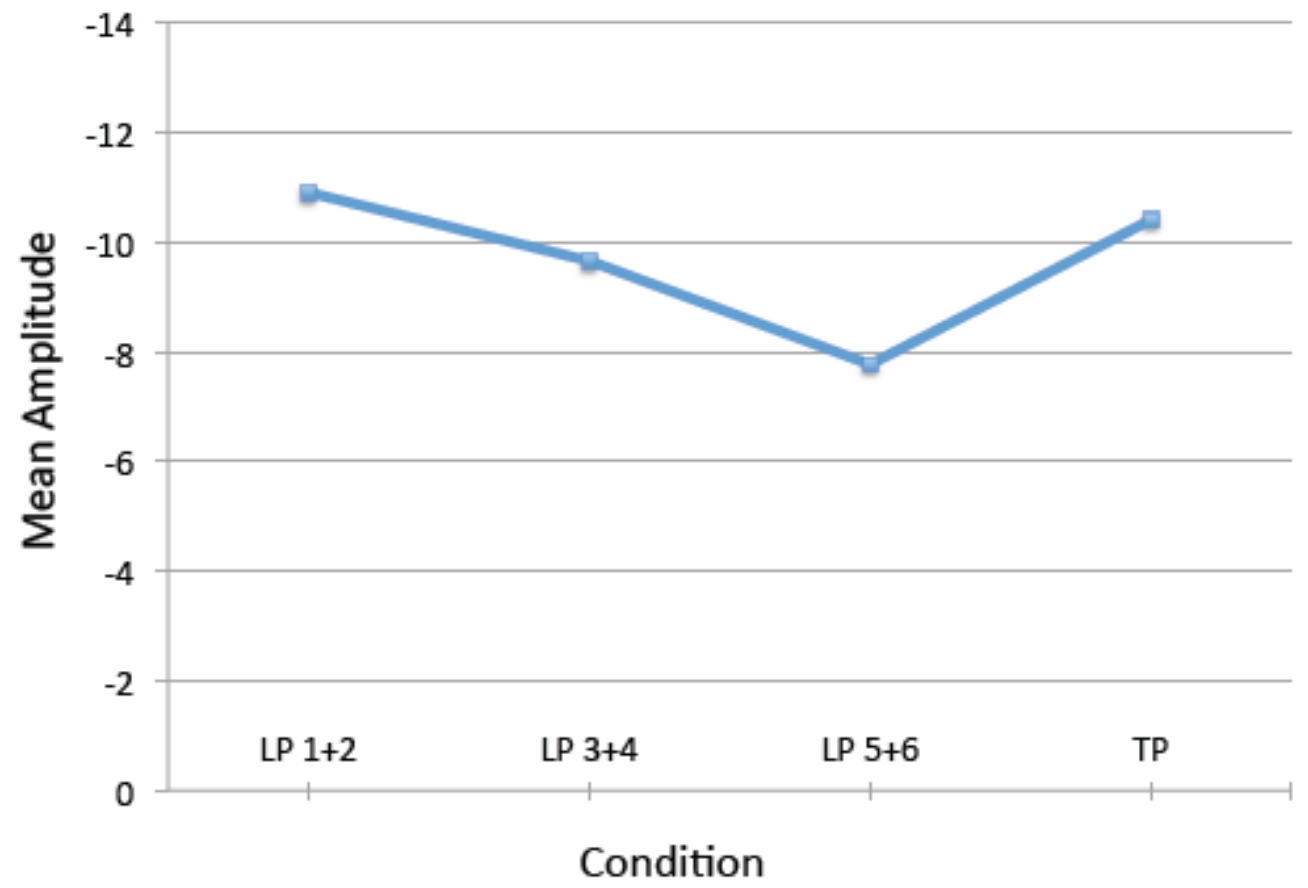

